\title{
Problèmes plaisans et délectables
}

Revue française d'automatique, d'informatique et de recherche opérationnelle. Recherche opérationnelle, tome 9, nº V2 (1975), p. 125.

<http://www.numdam.org/item?id=RO_1975_9_2_125_0>

(C) AFCET, 1975, tous droits réservés.

L'accès aux archives de la revue « Revue française d'automatique, d'informatique et de recherche opérationnelle. Recherche opérationnelle » implique l'accord avec les conditions générales d'utilisation (http://www.numdam.org/ legal.php). Toute utilisation commerciale ou impression systématique est constitutive d'une infraction pénale. Toute copie ou impression de ce fichier doit contenir la présente mention de copyright.

\section{Numdam}

Article numérisé dans le cadre du programme

Numérisation de documents anciens mathématiques

http://www.numdam.org/ 


\section{PROBLEMES PLAISANS ET DELEGTABLES}

\section{MARS 1972. - Pour jouer dans le métro}

Constituer des piles de jetons pour les désempiler au moindre coût c'est aussi affecter des rames de métro en fin de service à des voies de garage pour que leur sortie le lendemain exige le minimum de mouvements.

Même si la séquence des sorties est connue au moment des entrées le problème ne paraît pas simple.

\section{WITKOWSKI}

- $a$ et $b$ étant deux entiers premiers entre eux, quel est le plus grand entier $M(a, b)$ qui ne puisse être mis sous la forme $a p+b q, p$ et $q$ étant des entiers positifs.

- Quelle est la valeur de $M(39,53)$.

- Il existe un entier $e$ tel que tout $M(a, b)$ puisse être écrit $M(e, x)$.

C. W.

On a raconté que Von Neumann avait résolu de tête le problème suivant que vous connaissez sans doute :

Un curé et son vicaire croisent trois personnes au cours de leur promenade et le vicaire dit au curé « quel est l'âge de chacune de ces personnes sachant que le produit de ces âges est 2450 et que leur somme est égale à votre âge ».

Après un moment de réflexion le curé déclare " Il me manque une donnée ". « Je suis plus âgé que ces trois personnes » répond le vicaire.

Quel est l'âge du vicaire?

Mais que se passe-t-il si on remplace l'expression « 3 personnes" par « $n$ personnes »?

$$
\text { C. W. }
$$

